\title{
DIVERSIDAD VEGETAL Y USO ANTRÓPICO DE LOS PÁRAMOS DE SAMANGA (SECTORES ESPÍNDOLA Y EL TOLDO) Y SAN JUAN DE CACHIACO (CASERÍOS SAN JUAN Y TOTORA), AYABACA, PIURA
}

\author{
PLANT DIVERSITY AND ANTHROPIC USE IN SAMANGA PARAMO \\ (ESPÍNDOLA AND EL TOLDO SECTOR) AND IN SAN JUAN DE CACHIACO \\ PARAMO (SAN JUAN AND TOTORA SECTORS), AYABACA, PIURA
}

\author{
Ana Sabogal ${ }^{1}$ y Yakov Quinteros ${ }^{2}$
}

\begin{abstract}
Resumen
El artículo compara la flora de dos espacios: San Juan de Cachiaco y Samanga, ambos situados en los páramos de la provincia de Ayabaca, Piura. Ambos páramos corresponden al subpáramo. Se presenta la relación de plantas encontradas y endemismo. En el páramo de Samanga, se registraron 67 especies. Las familias más representativas fueron aquí: Asteraceae, Poaceae y Ericaceae. En el páramo de San Juan, se registraron 86 especies. Las familias más representativas fueron: Asteraceae, Rosaceae, Ericaceae y Cyperaceae. El páramo de San Juan es más húmedo y parece estar mejor conservado lo que se observa por la presencia de mayor cantidad de especies, familias, géneros y especies endémicas. Ambos páramos se encuentran amenazados por el pastoreo, estando el páramo de Samanga bajo mayor presión antrópica.

Palabras clave: Páramo, diversidad florística, uso antrópico, Ayabaca.
\end{abstract}

\begin{abstract}
The following article compares two habitats: the Samanga and San Juan de Cachiaco paramos in the province of Ayabaca. Both paramos could be classified as subparamo. Life forms and endemism are described. At Samanga paramo a total of 67 species were registered. The most representative families were: Asteraceae, Poaceae and Ericaceae. At San Juan Paramo, 86 species were registered. The most representative families were: Asteraceae, Rosaceae, Ericaceae and Cyperaceae. San Juan paramo from is more humid and better conserved; this is reflected in the number of family and species that were found . Both paramo are threatened by grazing, but Samanga paramo is under higher anthropic pressure.
\end{abstract}

Key words: Paramo, plants diversity, anthropic use, Ayabaca.

\section{Introducción.}

El páramo se define como un ecotono entre la línea de bosque y la línea de nieve que se encuentra ubicado en zonas húmedas y semihúmedas (Lauer, 1981). Los páramos están distribuidos en todas las montañas tropicales, pero en Sudamérica este ecosistema está mejor representado (Lauer, 1981), formando parches discontinuos de vegetación en alturas que oscilan entre 3000 y 5000 msnm y entre los grados 8 y 11 de latitud sur (Luteyn, 1992). Tanto en Asia como en el continente que se encuentra orientado hacia el Océano Pacifico, los páramos conforman parches de vegetación (Monasterio y Vuilleumier 1986 cit. Monasterio, 1998).

En el Perú los páramos están distribuidos a lo largo de las cadenas montañosas entre los 3000 y 3700 msnm al norte de la depresión de Huancabamba (Marcelo \& Millán, 2004). Estos se encuentran en las cuencas de los ríos Quiroz, Huancabamba y San Ignacio, es una zona de neblinas y suelos húmedos con abundante materia orgánica (Brack \& Mendiola,
2000; Proyecto Páramo Andino et al., 2009). Frey \& Lösch (2004), distinguen el páramo de la puna por su diferencia en el régimen de lluvias encontrando una marcada época de sequía y de helada para la puna, lo que la diferencia del páramo donde la neblina es constante. Los páramos del Perú han sido poco estudiados (Marcelo \& Millán, 2004).

Cabe preguntarse en qué medida existe una influencia de la depresión de Huancabamba para determinar el límite sur de los páramos en los Andes (Marcelo \& Millan, 2004). Este límite se debe probablemente al cambio de orientación de los Andes que juega un rol primordial en la distribución de la vegetación (Frey \& Lösch, 2004). El clima juega un rol primordial en la distribución y abundancia de la vegetación del páramo tanto en hábitat como en diversidad (Monasterio, 1998).

La flora del páramo está constituida por 112 familias, 479 géneros y alrededor de 3000 a 4000 especies de plantas vasculares (Luteyn et al., 1992). Las familias más frecuentes en los páramos son 
Asteraceae, Poaceae y Valerianaceae (Van der Hammen \& Cleef, 1986) y su distribución varía con la altura. En los páramos de Jaén (Cajamarca, Perú) las familias de dicotiledóneas más diversas son Asteraceae, seguida de Ericaceae, Melastomataceae, Scrophulariaceae, Campanulaceae y Rosaceae (Marcelo et al., 2006). Para los páramos de Samanga y San Juan de Cachiaco, provincia de Ayabaca, las familias más frecuentes son Asteraceae, Poaceae y Ericaceae (Sabogal, 2011).

Según la gradiente altitudinal Cuatrecasas (1958) dividió el páramo en tres zonas: el subpáramo, el páramo propiamente dicho y el superpáramo, las mismas que son mencionadas por Frey \& Lösch, (2004). El subpáramo se ubica entre los 3000 y los 3500 msnm y forma la transición entre el bosque andino y el páramo superior (Cuatrecasas, 1958; Cleef, 1978); en el dominan las familias Ericaceae y Asteraceae (Frey \& Lösch, 2004).

El páramo propiamente dicho, o páramo de pastos, se encuentra entre los 3500 y los 4300 msnm y está cubierto por pastos entre los que domina el género Calamagrostis y mezclado con arbustos enanos (Cleef, 1978). La vegetación se caracteriza por formar rosetas ramificadas, muchas de ellas de la familia Asteraceae; en sitios protegidos del viento, se encuentran pequeños matorrales y bosques aislados de Polylepis sp. (Rosaceae) (Cleef, 1978). Este páramo es marcadamente xerófilo, está compuesto por pastos en macollos dominados por Calamagrostis y Festuca o Chusquea (Luteyn, 1992) y es la zona de mayor endemismo del páramo (Luteyn, 1992). Cabe mencionar que hacia el norte, desde Perú hacia Ecuador, la zonación vegetacional varía.

El superpáramo, se sitúa entre los 4100 y los 4750 msnm, en el límite con la zona de la nieve y el hielo (Cleef, 1978). Aquí la vegetación es rala (Frey \& Lösch, 2004), discontinua y dispersa (Cleef, 1978). Los géneros característicos del superpáramo son: Draba, Lycopodium, Lachemilla, Poa y Agrostis, además de varios otros géneros de la familia Asteraceae y la presencia de líquenes y briofitas (Cleef, 1978).

De las 3000 a 4000 plantas vasculares del páramo, alrededor del $60 \%$ son plantas endémicas (Luteyn, 1992). En los páramos del Perú la familia Asteraceae presenta $40 \%$ de endemismo, Fabaceae $20 \%$, Poaceae 14\%, Orchidaceae 39\%, Melastomataceae 22\%, Rubiaceae 15\% y Solanaceae 24\% (Sagástegui et al., 2009). Otras familias menos frecuentes pero con elevado endemismo en el norte del Perú son: Bromeliaceae, Piperaceae con 37\%, Scrophulariaceae con 28\%, Lamiaceae con 27\% y Malvaceae (Sagástegui et al., 2009). El endemismo en el páramo es relativamente bajo a nivel de género pero muy alto a nivel de especie (Luteyn et al., 1992).

El páramo actualmente se encuentra amenazado por la presión antrópica, sobre todo debido al sobrepastoreo, y los impactos negativos repercuten sobre la vegetación. Se carece de estudios detallados de los cambios de vegetación que ello produce. Así mismo, los cambios de temperatura elevan la línea de vegetación de los páramos como consecuencia de los cambios climáticos globales; estos cambios no han sido documentados ya que para ello se requiere de largas series de tiempo.

Este estudio ofrece información sobre la diversidad florística de los páramos de Samanga (Sectores Espíndola y El Toldo) y San Juan de Cachiaco (caseríos San Juan y Totora), Ayabaca, Piura, comparando la diversidad florística y endemismo de ambos espacios. Los resultados servirán de línea base que permitirá realizar comparaciones futuras.

\section{Materiales y métodos.}

El páramo estudiado se encuentra en el departamento de Piura en la provincia y distrito de Ayabaca, sectores Espíndola y El Toldo (Comunidad de Samanga, predios de El Toldo y Espíndola) y en el distrito de Pacaipampa sector San Juan (Comunidad de San Juan de Cachiaco, predios de Totora, San Juan y Totora).

El área de estudio del páramo de Samanga está ubicada entre los 3207 y los 3356 msnm y entre las coordenadas geográficas $4^{\circ} 41^{\prime} 41^{\prime \prime} \mathrm{S}-79^{\circ} 28^{\prime} 56^{\prime \prime} \mathrm{O}$;

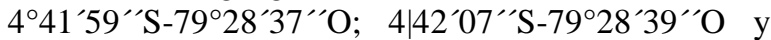
$4^{\circ} 41^{\prime} 33^{\prime \prime}-79^{\circ} 28^{\prime} 50^{\prime \prime} \mathrm{O}$. El espacio estudiado en el páramo de San Juan se ubica entre los 3168 y los 3227 msnm y entre las coordenadas geográficas $4^{\circ} 58^{\prime} 30^{\prime \prime}$ -

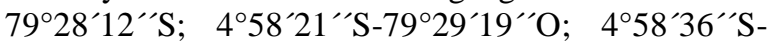
$79^{\circ} 28^{\prime} 21^{\prime \prime} \mathrm{O}$; 4 ${ }^{\circ} 58^{\prime} 20^{\prime \prime} \mathrm{S}-79^{\circ} 29^{\prime} 18^{\prime \prime} \mathrm{O}$.

Ambos espacios, el páramo de Samanga y el de San Juan, se encuentran a ocho horas del centro poblado más cercano a lomo de mula o a pie. La temperatura promedio del páramo de Pacaipampa, donde se ubica San Juan, está entre los 10 y los $16^{\circ} \mathrm{C}$ y la precipitación anual fluctúa entre 1900 y 2400 mm (CONDESAN, 2012). El páramo de Samanga, tiene una temperatura mínima de $8.1^{\circ} \mathrm{C}$ y máxima de $18.6^{\circ} \mathrm{C}$ y un promedio de precipitación de $1274 \mathrm{~mm}$ anuales (Proyecto Páramo Andino, Naturaleza y Cultura Internacional, Instituto de Montaña. 2009). La pendiente promedio de los espacios estudiados es de 17\% en San Juan y de $12 \%$ en Samanga. Los suelos del páramo poseen mucha materia orgánica y son muy porosos por lo que retienen mucha agua (Proyecto Páramo Andino, Naturaleza y Cultura Internacional, Instituto de Montaña. 2009).

Las colecciones se realizaron en los meses de Agosto, Octubre y Noviembre del 2009, durante una semana de cada mes. Sólo se consideraron plantas vasculares. Las especies colectadas fueron identificadas en el Herbario del Museo de Historia Natural de la Universidad Nacional Mayor de San Marcos (UNMSM). Todas las especies identificadas 
ya se encontraban en el herbario del museo razón por la cual si bien se las identificó y colocó el número de colecta no se depositaron en el herbario.

Las familias fueron ordenadas alfabéticamente, al igual que los géneros. Algunos especímenes sólo se identificaron a nivel de género por carecer de material reproductivo. Los nombres de las familias están organizados de acuerdo al Sistema de Clasificación de Cronquist (1981) según Mabberley (1989). Para la grafía de los nombres científicos se revisaron los trabajos de Brako y Zarucchi (1996) y The International Plant Name Index (IPNI, 2004). Para la determinación de las especies endémicas se utilizó el libro de Brako \& Zarucchi (1996).

\section{Resultados.}

Siguiendo la zonación vegetacional propuesta por Cuatrecasas (1958), en la zona de estudio se han podido reconocer dos zonas: el subpáramo y el páramo (páramo propiamente dicho). El subpáramo se caracteriza por encontrarse entre los 3000 y los 3500 msnm formando la transición entre el bosque andino y el páramo superior. Aquí se encuentran especies de Ericaceae y Asteraceae, mientras que a mayores altitudes se encuentran como dominantes arbustos enanos de Arcythophyllum nitidum (Rubiaceae) y Gaylussacia buxifolia (Ericaceae) mezclados con pastos (Cleef, 1978).

El páramo propiamente dicho, páramo medio, o páramo de pastos, se encuentra entre los 3500 y los 4300 msnm y este está cubierto principalmente por pastos del género Calamagrostis y rosetas ramificadas de la familia Asteraceae (Cleef, 1978). En este páramo medio, en el Perú como en los demás páramos, se encuentran pequeños matorrales de Hypericum sp. o de Senecio vaccinoides (Asteraceae) y bosques aislados de Polylepis sp. (Rosaceae) en los sitios protegidos del viento (Cleef, 1978). En este espacio se encuentran gran cantidad de plantas endémicas (Luteyn, 1992).

En el páramo de Samanga fueron colectadas 67 especies, 51 géneros y 25 familias. Del total de fanerógamas el $88.06 \%$ corresponden a dicotiledóneas, el 11.94\% a monocotiledóneas y el $1.49 \%$ a gimnospermas.

Aunque por su altitud el páramo de Samanga se encuentra en el subpáramo, por la presencia y dominancia marcada de hierbas en macollo corresponde a un páramo de pastos. Sin embargo, también existen plantas leñosas dispersas. Las hierbas encontradas fueron 39 (58.21\%), una de las cuales es trepadora (1.49\%). El total de plantas leñosas fue 27 (40.3\%) y de éstas 23 (34.33\%) son especies arbustivas y 4 (5.97\%) son árboles.

Dentro del total de fanerógamas, se encontraron 51 géneros pertenecientes a 25 familias (Tabla 1). Las 5 familias más diversas ordenadas en forma descendente fueron Asteracea (16 especies), Ericacea (7 especies), Poacea (6 especies), Apiaceae (4 especies) y Scrophulariaceae (4 especies) (Tabla 1). La distribución de familias según la localidad se puede observar en el Figura 1.

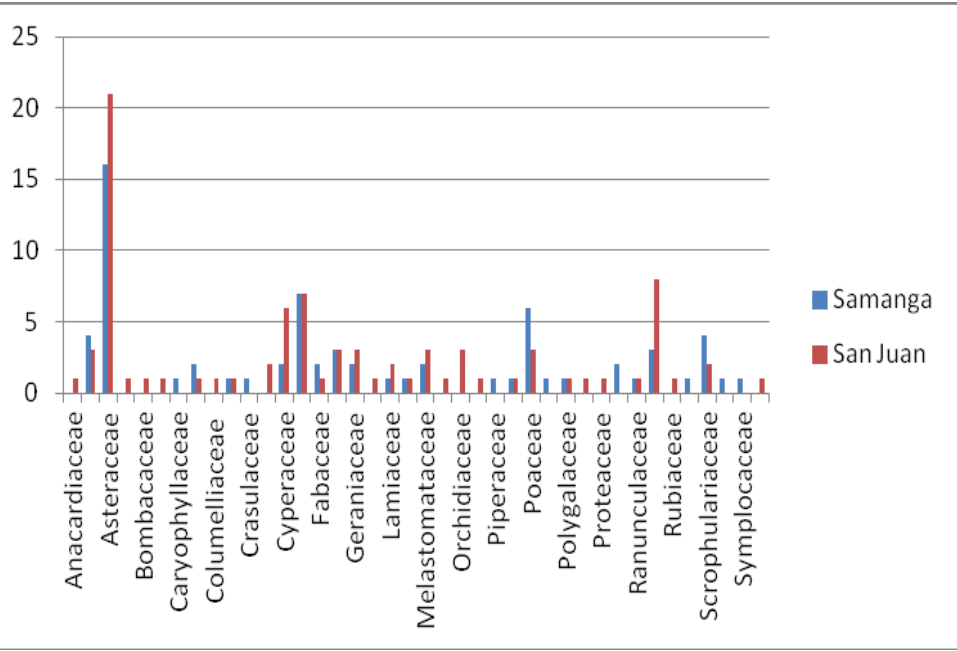

Figura 1. Distribución de familias en cada una de las localidades estudiadas.

El número total de especies endémicas encontradas para el lugar fue de 8 (11.94\%): de éstas el 50\% corresponden a la familia Asteraceae, $25 \%$ a la familia Gentianaceae y las demás familias con una sola especie (Tabla 2). Las especies que presentaron mayor frecuencia fueron: Jarava ichu Ruiz \& Pav (100\%), Orthrosanthus chimboracensis (Kunth) S.F. Baker (86.7\%), Vaccinium crenatum (D. Don ex Dunal) Sleumer (80\%), siendo las especies más abundantes Jarava ichu Ruiz \& Pav (51\%), Vaccinium crenatum (D. Don ex Dunal) Sleumer (7.2\%) y Brachyotum rostratum Triana (7\%). En la Figura 2 se observa la distribución de las especies endémicas en cada localidad.

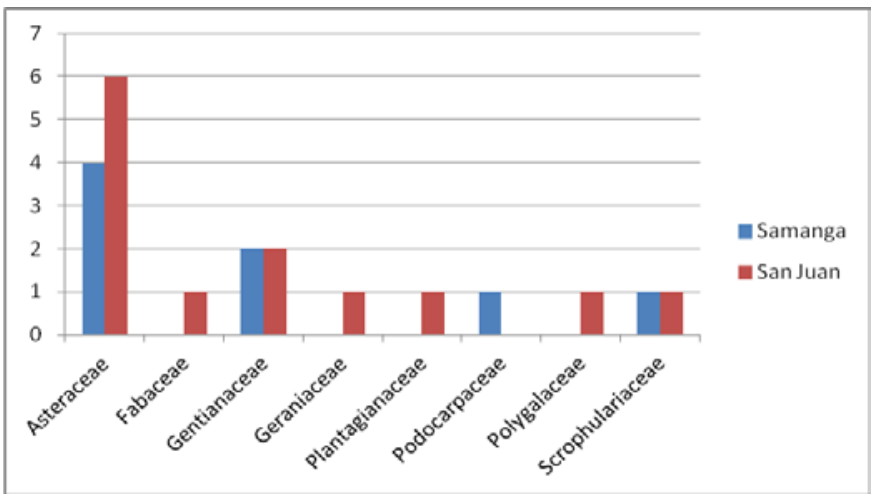

Figura 2. Endemismo en cada una de las localidades estudiadas. 
El páramo de San Juan es húmedo con presencia de lagunas y arroyos. En esta zona el paisaje está dominado por especies herbáceas con un porcentaje de $56.98 \%$, mientras que el $36.05 \%$ son especies arbustivas, $4.65 \%$ son árboles y sólo 2 especies son trepadoras. En San Juan se encontraron en total 86 especies entre angiospermas y gimnospermas, 67 géneros y 33 familias (Tabla 1). El 83.72\% son dicotiledóneas y 16.28\% monocotiledóneas. Las 4 familias más diversas ordenadas en forma decreciente fueron Asteraceae (21 especies), Rosaceae (8 especies), Ericaceae (7 especies) y Cyperaceae (6 especies). En el páramo de Samanga se encontró 58.21\% de especies herbáceas, $34.33 \%$ de especies arbustivas y 4.65\% de especies arbóreas; no se encontró ninguna especie trepadora. La distribución por estratos de la vegetación de ambas localidades se observa en la Figura 3.

Para todas las demás familias se encontraron 3 o menos especies (Tabla 1 y Figura 1). Además se registraron 14 especies endémicas, siendo Asteraceae la familia con mayor número de especies endémicas (Tabla 2). Las especies que presentaron mayor frecuencia fueron: Jarava cf. ichu Ruiz \& Pav. (96.66\%), Hypericum laricifolium Juss. (93.33\%) y Orthrosanthus chimboracensis (Kunth) S.F. Baker (70\%), siendo las especies con mayor abundancia Jarava cf. ichu Ruiz \& Pav. (32.17\%), Sphagnum sp. L (9.93\%) y Hypericum laricifolium Juss (7.08\%).

La similitud entre ambas comunidades se midió mediante el Índice de Sorensen, el cual fue de tan solo 26,09\%, siendo el número de especies comunes 27.

\section{Discusión.}

Teniendo en cuenta la altitud (Cleef, 1978) y las familias vegetales, Ericaceae y Asteraceae, el espacio estudiado se ubica en el subpáramo (Cleef, 1978; Frey \& Lösch, 2004), en la transición entre el bosque andino y el páramo superior (Cleef, 1978). Así mismo los géneros presentes como Gynoxis, Vaccinium, Miconia y Hesperomeles son característicos del subpáramo (Luteyn, 1992).

Si bien en ambos espacios dominan las plantas herbáceas $(56.98 \%$ en San Juan y 58.21\% en Samanga), el porcentaje de plantas leñosas llama la atención llegando a ser de $40.7 \%$ para San Juan (4.65\% árboles y $36.05 \%$ arbustos) y de $40.3 \%$, para Samanga (5.97\% árboles y $34.33 \%$ arbustos).

En el páramo de Samanga se puede observar la especie Podocarpus glomeratus, conífera que usualmente crece en los bosques húmedos; su presencia aglomerada en algunas zonas podría indicar que algunas áreas fueron bosques y debido a la presencia antrópica (poblados asentados alrededor del páramo) y la consiguiente deforestación y sobrepastoreo, se transformaron en pajonales, lo que

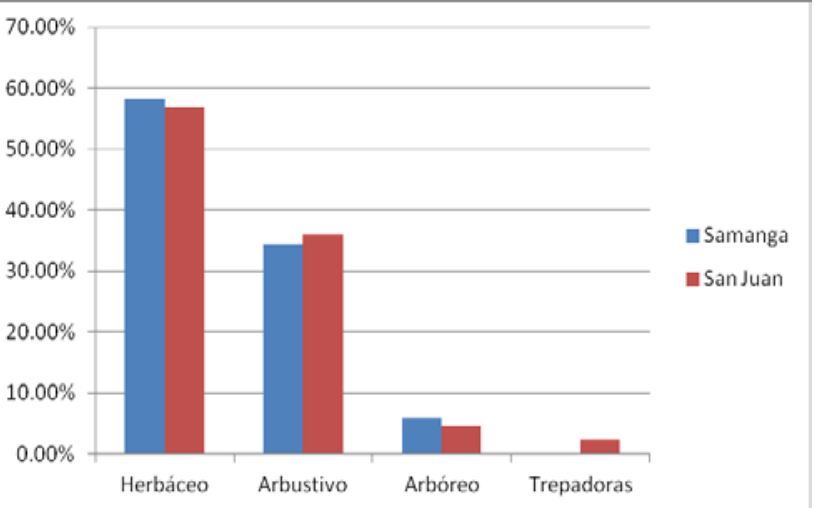

Figura 3. Distribución por estratos de la vegetación.

se refuerza si se tiene en cuenta que aquí la diversidad es considerablemente menor que en San Juan.

En ambos espacios, tanto en San Juan como en Samanga, se encontró presencia dominante de las familias Asteraceae y Ericaceae, familias características del subpáramo (Cleef, 1978, Frey \& Lösch, 2004). Cabe resaltar que de los géneros registrados para el norte del Perú, la más alta diversidad se encuentra en la familia Asteraceae, con 176 géneros y 754 especies (Sagástegui et al., 2009). En cambio los géneros de mayor distribución encontrados en los páramos ecuatorianos son Valeriana, Calamagrostis, Werneria y Gentianella (Sklenár \& Jorgensen, 1999), todos ellos presentes también en la zona de estudio.

Los géneros característicos en pendientes para los páramos de Ecuador son Hypericum, Diplostephium, Pentacalia, Penettya, Valeriana, Acaena, Werneria, Halenia, Gentiana y Gentianella (Luteyn, 1992). En el páramo de San Juan, donde la pendiente es alrededor de $17 \%$, se encontraron 7 (Hypericum, Diplostephium, Pentacalia, Valeriana, Halenia, Gentiana y Gentianella) de los 10 géneros mencionados por Luteyn (1992) para estos espacios. En Samanga, en cambio, donde la pendiente es alrededor de $12 \%$, se encontraron sólo 5 (Werneria, Hypericum, Halenia, Gentiana y Gentianella).

La humedad de este páramo y su cercanía a las lagunas se refleja en la presencia del género Plantago en ambos espacios (Rauh, 1979) y especies como Scirpus caespitosus y Lachemilla orbicullata, características de zonas húmedas (Brako \& Zarucchi, 1996). Por las características descritas para cada lugar, mencionadas líneas arriba, se nota una gran diferencia entre los promedios de precipitación de los dos espacios estudiados. La precipitación anual fluctúa entre 1900 y $2400 \mathrm{~mm}$ para San Juan (CONDESAN, 2012), mientras que el promedio de precipitación para el páramo de Samanga es de $1274 \mathrm{~mm}$ anuales (Marcelo et al., 2009). Si bien no se observa gran diferencia entre las especies más frecuentes, siendo para ambos espacios la especie con mayor frecuencia y abundancia Jarava ichu Ruiz \& Pav., las diferencias 
entre ambos espacios se refleja en la abundancia de las especies.

En el páramo de la zona de estudio el porcentaje de plantas endémicas es bajo (14.28\% en San Juan y sólo $11.94 \%$ en Samanga), si se considera que cerca del 60\% las plantas de los páramos son endémicas (Luteyn, 1992). Si se analiza el endemismo para cada familia se observa que para ambos espacios estudiados el 50\% de las especies endémicas corresponden a la familia Asteraceae lo que coincide con Sagastegui et al. (2009). También en los páramos de Cajamarca los géneros endémicos se concentran en la familia Asteraceae (Marcelo et al, 2006). En el páramo de Ecuador, las familias que presentan elevado endemismo son Orchidaceae y Asteraceae (MenaVáscones, 2011). Cabe resaltar que en los páramos estudiados no se encontró ninguna planta de la familia Orchidaceae. La segunda familia con mayor endemismo es Gentianaceae (25\% Samanga y 21\% San Juan). La presencia de mayor endemismo y mayor diversidad en los páramos de San Juan podría indicar una menor alteración del espacio.

El Índice de Sorensen indica que la similitud entre ambos espacios es tan sólo del $26.09 \%$ por lo que se puede confirmar que se trata de dos comunidades diferentes.

\section{Conclusiones.}

Los páramos estudiados de Samanga y San Juan se encuentran en el subpáramo, lo que se concluye por la altitud y las especies encontradas. La familia que presentó mayor frecuencia y abundancia fue Asteraceae. En el páramo de Samanga, las familias más representativas fueron: Asteraceae, Poaceae y Ericaceae. En el páramo de San Juan las familias más representativas fueron: Asteraceae, Rosaceae, Ericaceae y Cyperaceae. En ambas localidades domina la especie Jarava ichu Ruiz \& pav. Llama la atención la elevada presencia de especies arbóreas, mayor al 40\% para ambos espacios. En el páramo de Samanga la presencia de Podocarpus glomeratus parece indicar la presencia de bosques que fueron deforestados o sobrepastoreo, transformándose en pajonales.

En el páramo de ambas zonas de estudio el porcentaje de plantas endémicas es muy bajo (14.28\% en San Juan y $11.94 \%$ en Samanga). Para ambas localidades el endemismo se concentra a nivel de la familia Asteraceae. El páramo de Samanga está fuertemente alterado por actividades antrópicas lo que se refleja en una menor diversidad y endemismo.

Los espacios estudiados corresponden a dos comunidades diferentes, ya que la similitud entre ambos espacios es tan solo de $26.09 \%$.

\section{Agradecimientos.}

A la población de Espíndola, San Juan y El Toldo, a Naturaleza y Cultura Internacional, al Mountain Institute por el apoyo logístico y a la Pontificia
Universidad Católica del Perú por el apoyo brindado para realizar este trabajo .

Esta publicación ha sido posible con la financiación del Programa de Becas del Proyecto Páramo Andino del CIP-CONDESAN.

\section{Literatura citada.}

Brack A. \& Mendiola C. 2000. Ecología del Perú. Bruño. Lima. 495 p.

Brako L. \& Zarucchi J. 1996. Catálogo de las angiospermas y gimnospermas del Perú. Missouri Botanical Garden. $1286 \mathrm{p}$.

Cleef A. M. 1978. Characteristics of neotropical paramo vegetation and its subantarctic relations. En:

Erdwissenschaftlicge Forschung vol. XI. Franz Steiner ed. Wiesbaden. pp. 356-390.

CONDESAN. 2012. Características Ambientales. http://www.condesan.org/ppa/node/2537.

Cronquist, A. 1981. An Integrated System of Classification of Flowering Plants. Columbia University Press.

Cuatrecasas J. 1958. Aspectos de la vegetación natural de Colombia. Revista de la Academia Colombiana de Ciencias Exactas y Físicas. 10 (40). pp: 221-264.

Frey W. \& Lösch, R. 2004. Lehrbuch der Geobotanik. Ed. Elsevier. $2^{\circ}$ Ed. München. 528 p.

IPNI. 2004. The International Plant Name Index. http://www.ipni.org/ipni/idAuthorSearch.do? id=83491\&show_history=false\&output_format=normal [revisado Noviembre 2010]

Keating P. 1999. Changers in páramo vegetation along and elevation gradient in southern Ecuador. Journal of the Torrey Botanical Society 126 (2). pp: 159- 175.

Lauer W. 1981. Ecoclimatological conditions of the paramo belt in the tropical high mountains. Mountain Research and Development, Vol. 1, N³-4. pp: 209-221.

Luteyn J.L. 1992. Paramo: Why study them? En: Páramo An Andean Ecosystem under Human Influence. Edited by: Balslev, H \& Luteyn, J.L.; Academic Press.p.1-14.

Luteyn J.L. Cleef, A.M. \& Rangel Ch., O. 1992. Plant diversity in paramo: Towards a checklist of páramo plants and generic flora. En: Paramo An Andean Ecosystem under Human Influence. Edited by: $\mathrm{H}$. Balslev \& Luteyn; Academic Press. p.71-84.

Mabberley D. J. 1989. The Plant-book. Cambridge University Press. Cambridge. 707 pp.

Marcelo J. \& Millán J. 2004. Estudio de la diversidad florística del páramo sectores: El Espino y Palmbe, distrito de Sallique, Provincia de Jaén. Tesis para optar el título de Ingeniero Forestal. Universidad Nacional de Cajamarca-Sección Jaén.

Marcelo J., Sánchez I., Millán J. 2006. Estado actual de la diversidad florística del Páramo sectores: El Espino y Palambe, Sallique, Jaén. Cajamarca. Perú. Ecol. apl. Vol. 5 pp. 1-8.

Mena-Váscones, P. 2011. La biodiversidad de los páramos en el Ecuador. http://www.banrepcultural.org/blaavirtual/geografia/con gresoparamo/la-biodiversidad.pdf. pp. 496-513.

Monasterio M. 1998. Evolución y transformación de los páramos en la cordillera de Mérida: Paisajes naturales y culturales. En: paisajes culturales de los Andes. Reunión de expertos, Chivay, Arequipa.

Proyecto Páramo Andino, Naturaleza y Cultura Internacional, Instituto de Montaña. 2009. El 
Diagnóstico Socio-Ecológico (DSE) del Plan de Manejo Participativo del Páramo. Vol.1. La realidad del Páramo de Samanga. Ayabaca, Piura, Perú. Pp: 84.

Rauh W. 1979. Perú país de los contrastes. Separata del Boletín de Lima. Vol. 1 \& 2. p: 21-24.

Sabogal A; Roca F.; Quinteros Y; Watson A; Yepes A \& Quiñe E. 2011. Guía de plantas de la Comunidad de Samanga (sectores El Toldo y Espíndola) y del predio San Juan de Cachiaco (Caseríos San Juan y Totora). Lima, Instituto de Montaña, Proyecto páramo andino.

Sagastegui, A, Dillon M, Sánchez, I., González S. \& Asencio P. 2009. Diversidad florística del norte del Perú. En: http://www.sacha.org/envir/peru/peru_sp.htm revisado 06.10.2009.
Sklenár P. \& Jorgensen P. M. 1999. Distribution of páramo plants in Ecuador. Journal of Biogeography. Vol. 26 N4. Pp: 681-691.

Van der Hammen T \& Cleef A.M. 1986. Development of the high Adean páramo flora and vegetation. En: Vuilleumier, F \& Monasterio, M., High altitude tropical biogeography. Oxford University Press. New York. Pp: 153-201.

Van der Hammen T, Werner J. H. \& van Dommelen $H$. 1973. Palynological record of the upheaval of the Northen Andes: a study of the Pliocene and Lower Quaternary of the Columbian Eastern Cordillera and the early evolution of its High-Andean biota. Review Palaeobotany and Palynology. Vol 16. pp: 1-122.

Tablas

Tabla 1. Lista florística de los páramos de los sectores Samanga y San Juan.

\begin{tabular}{|c|c|c|c|c|}
\hline $\mathrm{N}^{\circ}$ & Nombre científico & Familia & $\begin{array}{c}\text { Sector } \\
\text { Samanga } \\
\text { y Ncolecta }\end{array}$ & $\begin{array}{c}\text { Sector } \\
\text { San Juan } \\
\text { y N } \mathrm{N}^{\circ} \text { colecta }\end{array}$ \\
\hline 1 & Mauria simplicifolia Kunth & Anacardiaceae & & 1122 \\
\hline 2 & Azorella multifida Pers. & Apiaceae & & 1096 \\
\hline 3 & Azorella crenata Pers. & Apiaceae & & 1097 \\
\hline 4 & Oreomyrrhis andicola Endl & Apiaceae & 1028 & \\
\hline 5 & Hydrocotyle alchemilloides A.Rich. & Apiaceae & 1013 & \\
\hline 6 & Hydrocotyle aff. peruviana Wolff & Apiaceae & 1023 & \\
\hline 7 & Eryngium humile Cav. & Apiaceae & 982 & 1058 \\
\hline 8 & Senecio usgorensis Cuatrec. & Asteraceae & & 1145 \\
\hline 9 & Senecio sp. L. & Asteraceae & & 1119 \\
\hline 10 & Senecio culcitioides Sch.Bip. & Asteraceae & & 1130 \\
\hline 11 & Pentacalia andicola (Turcz.) Cuatrec. & Asteraceae & & 1144 \\
\hline 12 & Oritrophium repens (Kunth) Cuatrec. & Asteraceae & & 1133 \\
\hline 13 & Munnozia senecionidis Benth. & Asteraceae & & 1132 \\
\hline 14 & Loricaria ferruginea Wedd. & Asteraceae & & 1084 \\
\hline 15 & Hieracium leptocephalum Benth. & Asteraceae & & 1146 \\
\hline 16 & Gynoxys calyculisolvens Hieron. & Asteraceae & & 1070 \\
\hline 17 & Gamochaeta sp. Wedd. & Asteraceae & & 1085 \\
\hline 18 & Diplostephium meyenii Wedd. & Asteraceae & & 1142 \\
\hline 19 & Diplostephium foliosissimum S.F.Blake & Asteraceae & & 1141 \\
\hline 20 & Chaptalia oblonga D.Don & Asteraceae & & 1140 \\
\hline 21 & Baccharis latifolia Pers. & Asteraceae & & 1093 \\
\hline 22 & Baccharis cf. caespitosa Pers. & Asteraceae & & 1129 \\
\hline 23 & Ageratina piurae (B.L.Rob.) R.M.King \& H.Rob. & Asteraceae & & 1131 \\
\hline 24 & Ageratina pentlandiana (DC.) R.M.King \& H.Rob. & Asteraceae & & 1139 \\
\hline 25 & Werneria nubigena Kunth & Asteraceae & 1016 & \\
\hline 26 & Stevia cf. andina B. Robinson & Asteraceae & 1009 & \\
\hline 27 & Senecio llewelynii Cuatrec. & Asteraceae & 951 & \\
\hline
\end{tabular}


28 Senecio aff. evacoides

29 Paranephelius ovatus Wedd.

30 Hypochaeris graminea Hieron.

31 Chuquiraga weberbaueri Tovar

32 Chuquiraga aff. spinosa

33 Baccharis tricuneata Pers.

34 Baccharis sp.

35 Baccharis obtusifolia Kunth

36 Baccharis genistelloides (Lam.) Pers

37 Asteracea indeterminada

38 Ageratina wurdackii R.M.King \& H.Rob.

39 Ageratina sp. O.Hoffm.

40 Achyrocline alata DC.

41 Berberis lutea Ruiz \& Pav.

42 Eriotheca ruizii (K.Schum.) A.Robyns

43 Bromelia sp. ex L.

44 Cerastium subspicatum Wedd.

45 Hypericum andinum Gleason

46 Hypericum laricifolium Juss.*

47 Columellia oblonga Ruiz \& Pav.

48 Dichondra microcalyx (H. Hall) Fabris

49 Echeveria sp.

50 Weinmannia sp. Ruiz \& Pav.

51 Weinmannia microphylla Ruiz \& Pav.

52 Scirpus cf. caespitosus Willd. ex Kunth

53 Cyperus prolixus Balb. ex Kunth

54 Carex sp. L.

55 Carex polystachya Sw. ex Wahlenb.

56 Becquerelia cymosa Kunth

57 Oreobolus goeppingeri Suess.

58 Cyperaceae indeterminada

59 Bejaria mathewsii Field. \& Gardn.

60 Vaccinium floribundum Kunth

61 Vaccinium crenatum (D.Don exDunal) Sleumer

62 Thibaudia sp. Ruiz \& Pav.

63 Gaultheria reticulata Kunth

64 Gaultheria buxifolia Willd.

65 Disterigma sp. Nied.

66 Bejaria aestuans L.

67 Lupinus peruvianus Ulbr.

68 Trifolium cf. peruvianum Vogel

69 Lupinus sp.

70 Gentianella bicolor (Wedd.) Fabris ex J.S.Pringle

\begin{tabular}{|c|c|c|}
\hline Asteraceae & 961 & \\
\hline Asteraceae & 1047 & \\
\hline Asteraceae & 952 & 1028 \\
\hline Asteraceae & 1040 & \\
\hline Asteraceae & 984 & \\
\hline Asteraceae & 967 & \\
\hline Asteraceae & 1006 & \\
\hline Asteraceae & 983 & 1112 \\
\hline Asteraceae & 992 & \\
\hline Asteraceae & 990 & \\
\hline Asteraceae & 979 & \\
\hline Asteraceae & 973 & 1065 \\
\hline Asteraceae & 966 & 1083 \\
\hline Berberidaceae & & 1075 \\
\hline Bombacaceae & & 1121 \\
\hline Bromeliaceae & & 1092 \\
\hline Caryophyllaceae & & 1108 \\
\hline Clusiaceae & 1051 & \\
\hline Clusiaceae & 945 & 1067 \\
\hline Columelliaceae & & 1101 \\
\hline Convolvulaceae & 983 & 1099 \\
\hline Crasulaceae & 981 & \\
\hline Cunnoniaceae & & 1143 \\
\hline Cunnoniaceae & & 1153 \\
\hline Сyperaceae & & 1061 \\
\hline Сyperaceae & & 1062 \\
\hline Сурегасеае & & 1114 \\
\hline Сyperaceae & & 1149 \\
\hline Суреraceae & & 1148 \\
\hline Суреraceae & 1031 & 1115 \\
\hline Сyperaceae & 947 & \\
\hline Ericaceae & & 1071 \\
\hline Ericaceae & 1005 a & 1157 \\
\hline Ericaceae & 1018 & \\
\hline Ericaceae & 972 & 1100 \\
\hline Ericaceae & 971 & 1064 \\
\hline Ericaceae & 987 & 1156 \\
\hline Ericaceae & 978 & 1155 \\
\hline Ericaceae & 977 & 1063 \\
\hline Fabaceae & & 1057 \\
\hline Fabaceae & 1026 & \\
\hline Fabaceae & 975 & \\
\hline
\end{tabular}




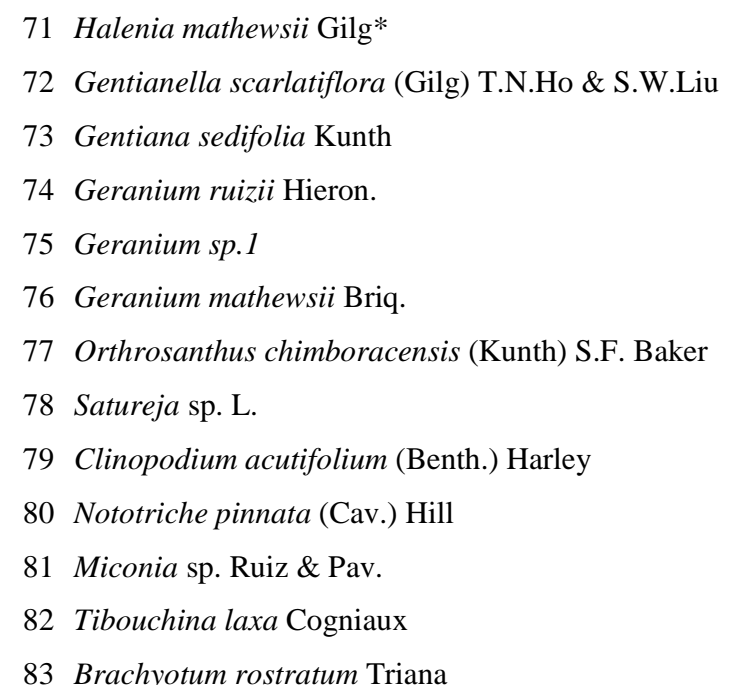

83 Brachyotum rostratum Triana

84 Epilobium sp. Dill. ex L.

85 Stelis sp. Sw.

86 Epidendrum sp. L.

87 Epidendrum hemiscleria Rchb.f.

88 Oxalis sp. L.

89 Peperomia galioides Kunth

90 Plantago lamprophylla Pilg.

91 Plantago lanceolata Hook.

92 No determinada

93 Jarava cf. ichu Ruiz \& Pav.

94 Vulpia sp. C.C.Gmel.

95 Paspalum sp. L.

96 Muhlenbergia sp. Schreb.

97 Festuca sp. L.

98 Calamagrostis minima ( Pilg. ) Tovar

99 Agrostis sp. L.

100 Podocarpus glomeratus Don in Lamb.

101 Monnina tomentella Chodat

102 Polygala sp. L.

103 Muehlenbeckia aff. hastulata I.M.Johnst.

104 Oreocallis mucronata (Willd. ex Roem. \& Schult.) Sleumer

105 Oreocallis grandiflora R.Br.

106 Ranunculus aff flagelliformis Sm.

107 Rubus megalococcus Focke

108 Rosa sp. L.

109 Polylepis weberbaueri Pilg.

110 Lachemilla sp. Rydb.

111 Lachemilla barbata (Presl) Rothm.

112 Hesperomeles heterophylla Hook.

113 Hesperomeles ferruginea Lindl.

\begin{tabular}{|c|c|c|}
\hline Gentianaceae & 1033 & 1066 \\
\hline Gentianaceae & 958 & \\
\hline Gentianaceae & 1004 & 1113 \\
\hline Geraniaceae & & 1091 \\
\hline Geraniaceae & 1020 & 1074 \\
\hline Geraniaceae & 950 & 1072 \\
\hline Iridaceae & & 1134 \\
\hline Lamiaceae & & 1076 \\
\hline Lamiaceae & 995 & 1102 \\
\hline Malvaceae & 985 & 1104 \\
\hline Melastomataceae & & 11201147 \\
\hline Melastomataceae & 964 & 1106 \\
\hline Melastomataceae & 1010 & $1078,1105$. \\
\hline Onagraceae & & 1103 \\
\hline Orchidiaceae & & 1082 \\
\hline Orchidiaceae & & 1081 \\
\hline Orchidiaceae & & 1080 \\
\hline Oxalidaceae & & 1111 \\
\hline Piperaceae & 974 & \\
\hline Plantaginaceae & & 1055 \\
\hline Plantaginaceae & 1050 & \\
\hline Poaceae & & 1059 \\
\hline Poaceae & & 1118 \\
\hline Poaceae & 959 & \\
\hline Poaceae & 948 & \\
\hline Poaceae & 1053 & \\
\hline Poaceae & 981 & 1117 \\
\hline Poaceae & 962 & \\
\hline Poaceae & 1054 & \\
\hline Podocarpaceae & 946 & \\
\hline Polygalaceae & & 1073 \\
\hline Polygalaceae & 956 & \\
\hline Polygonaceae & & 1098 \\
\hline Proteaceae & 1055 & \\
\hline Proteaceae & 963 & 1060 \\
\hline Ranunculaceae & 960 & 1107 \\
\hline Rosaceae & & 1069 \\
\hline Rosaceae & & 1079 \\
\hline Rosaceae & & 1077 \\
\hline Rosaceae & & 1124 \\
\hline Rosaceae & & 1073 \\
\hline Rosaceae & & 1123 \\
\hline Rosaceae & & 1127 \\
\hline
\end{tabular}




\begin{tabular}{|c|c|c|}
\hline Rosaceae & 953 & \\
\hline Rosaceae & 1033 & \\
\hline Rosaceae & 1001 & $1068,1109$. \\
\hline Rubiaceae & & 1094 \\
\hline Santalaceae & 968 & \\
\hline Scrophulariaceae & & 1095 \\
\hline Scrophulariaceae & 1007 & 1126 \\
\hline Scrophulariaceae & 996 & \\
\hline Scrophulariaceae & 976 & \\
\hline Scrophulariaceae & 1029 & \\
\hline Solanaceae & 970 & \\
\hline Symplocaceae & 980 & \\
\hline Valerianaceae & & 1125 \\
\hline
\end{tabular}

*aff. En Samanga

$+c f$. en Samanga

Tabla 2. Especies endémicas encontradas.

\begin{tabular}{|c|c|c|c|}
\hline Familia & Especie & Samanga & San Juan \\
\hline Asteraceae & Ageratina wurdackii R. M. King \& H. Rob. & $\mathrm{x}$ & $\mathrm{x}$ \\
\hline Asteraceae & Bracharis latifolia Pers. & & $\mathrm{x}$ \\
\hline Asteraceae & Chuquiraga spinosa (R \&P) D. Don & $\mathrm{x}$ & \\
\hline Asteraceae & Chuquiraga weberbaueri Tovar & $\mathrm{x}$ & \\
\hline Asteraceae & Diplostephium foliosissimum S. F. Blake & & $\mathrm{x}$ \\
\hline Asteraceae & Gynoxis calyculisolvens Hieron. & & $\mathrm{x}$ \\
\hline Asteraceae & Loricaria furruginea Wedd. & & $\mathrm{x}$ \\
\hline Asteraceae & Senecio llewelynii Cuaterc. & $\mathrm{x}$ & \\
\hline Asteraceae & Senecio usgorensis Cuatrec. & & $\mathrm{x}$ \\
\hline Fabaceae & Lupinus peruvianus Ulbr. & & $\mathrm{x}$ \\
\hline Gentianaceae & Gentianella bicolor (Wedd.) Fabris ex. J. S. Pringle & & $\mathrm{x}$ \\
\hline Gentianaceae & Gentianella scarlatiflora (Gilg) T. N. Ho \& S. W. Liu & $\mathrm{x}$ & \\
\hline Gentianaceae & Halenia mathewsii Gilg* & $\mathrm{x}$ & $\mathrm{x}$ \\
\hline Geraniaceae & Geranium ruizii Hieron. & & $\mathrm{x}$ \\
\hline Plantagianaceae & Plantago lamprophylla Pilg. & & $\mathrm{x}$ \\
\hline Podocarpaceae & Podocarpus glomeratus Don in Lamb. & $\mathrm{x}$ & \\
\hline Polygalaceae & Monina tomentella Chodat. & & $\mathrm{x}$ \\
\hline Scrophulariaceae & Bartsia crisafulli N. Holmgrem & $\mathrm{x}$ & \\
\hline Scrophulariaceae & Castilleja fissifolia L. F. & & $\mathrm{X}$ \\
\hline
\end{tabular}

*aff. En Samanga

\footnotetext{
${ }^{1}$ Instituto de Ciencias de la Naturaleza, Territorio y Energías Renovables de la Pontificia Universidad Católica del Perú (INTE-PUCP). Av. Universitaria 1801, San Miguel, Lima, Perú. asabogal@pucp.edu.pe.

${ }^{2}$ Departamento de Etnobotánica, Museo de Historia Natural, Universidad Nacional Mayor de San Marcos. Av. Arenales 1256, Jesús María, Lima, Perú. yacov281@hotmail.com.
} 